\section{Krukenberg's chopsticks}

The Krukenberg operation is named after the German surgeon who first suggested that a forearm stump could be made into a functional limb by separating the radius and ulna and so providing the patients with a pair of muscle-powered pincers. Sixty years on the procedure is a good example of a simple practical operation that is sufficiently rare in civilian practice to merit occasional readvertisement. It is, sadly, also one of the long line of medical innovations born out of war and bred by the continuing effects of military strife. Guerrilla fighters learning to use grenades or crawling through antipersonnel minefields may forfeit their hands and sight. Bangladesh and Vietnam have provided the most recent crops of these injuries in young men, and their rehabilitation has recently been reviewed. ${ }^{12}$

Surgeons who use the Krukenberg operation admit the ugliness of the functioning pincers, but they counter this by pointing out that such a stump does not preclude wearing a cosmetic prosthesis-and may even improve the management of a functional one. Furthermore, if the victim is blind then the sensory feedback from the Krukenberg stump will be invaluable. Even sighted amputees find many practical benefits from having a functional stump with sensation. American orthopaedic surgeons, who made substantial contributions to this subject in Vietnam, have filmed bilateral Krukenberg amputees performing many difficult tasks with these useful stumps, including the expert use of chopsticks.

Though most candidates for the Krukenberg operation come from military sources, patients do lose their hands in industrial accidents, which are becoming more frequent in developing countries. Workers on the land learning to use powered agricultural machinery have a high accident rate, while the proliferation of light industrial plants in villages is also creating more work for the surgeons. When both arms are damaged any procedure, however bizarre in appearance, will be welcome if it provides prehension and tactile sensation without the investment of time and money required for the fitting of a conventional prosthesis.

In developed societies such as Britain orthopaedic surgeons are only too familiar with the cable-operated split-hook or "functional" hand which is kept in the cupboard and dusted off for occasional parade to the doctor. The single upper limb amputee tends to survive in the Welfare State by a mixture of dependence on the remaining limb and disability registration. Wheelchairs may provide an irreversible disincentive to lower limb amputees to relearn mobility, and there is a similar parallel whereby television may give a passive alternative to more active hobbies for forearm amputees in the privacy of their own homes. Yet patients can use such beginnings to acquire the confidence to use a versatile stump for more gainful pursuits, and a Krukenberg procedure might bring some twohanded tasks within the grasp of single-arm amputees.

These two papers offer good bibliographies and useful technical advice. Both authorities emphasise the importance of a careful myoplastic approach, with gentle separation and reinsertion of radially and ulnarly disposed muscles to their respective bones. If skin grafting of the interosseous cleft can be avoided, much better sensation is provided for the opposing surfaces, and Nathan and Trung ${ }^{2}$ describe in detail how to excise the correct amount of muscle, so allowing primary closure. The minimum recommended length of stump is $8 \mathrm{~cm}$ from the insertion of the biceps; the optimum is $12 \mathrm{~cm}$ or more. This procedure seems likely to be of great practical value in developing countries for the rest of this century; and it is not impossible that a "Krukenberg Klub" may succeed "McIndoe's guinea-pigs" in achieving cosmetic acceptability and restored function in more affluent societies.

1 Garst, R J, The Disabled in Developing Countries. Proceedings of a Symposium on Appropriate Technology and Delivery of Health and Welfare Services for the Disabled in Developing Countries, p 16. London, Commonwealth Foundation, 1977.

2 Nathan, P A, and Trung, N B, fournal of Hand Surgery, 1977, 2, 127.

\section{Testing monocyte function}

The monocyte-macrophage system is of critical importance to host defence. It begins in the bone marrow with the differentiation of the monoblast from precursor stem cell lines and continues with the differentiation of the mature peripheral blood monocyte, which later transforms into the tissue macrophage.

The connection between the peripheral blood monocyte and the tissue macrophage was suggested by the classical studies of Metchnikoff ${ }^{3}$ and Aschoff ${ }^{2}$ and later shown conclusively by Lewis $^{3}$ and Ebert and Florey. ${ }^{4}$ Since these early studies experimental pathologists have concentrated their attention on the tissue macrophage, which in its "activated" form is the most important functional part of the host's defence system, and the blood monocyte has been neglected. This and the fact that the blood monocyte is a short-lived transition cell help to explain why monocyte function tests have been slow to develop. Nevertheless, we now know that the monocyte is a cell with a highly developed phagocytic potential of great practical importance in man and in most mammals. It is the major defence system against intracellular pathogenic bacteria, fungi, and various intracellular parasites; it plays a part in antigen processing; and it interacts with lymphoid cells in cellmediated immunity. Recent evidence suggests that the monocyte may provide a defence system against some tumours, ${ }^{56}$ and in addition that it may be the source of the colony-stimulating activity required for granulopoiesis. ${ }^{78}$

The development of granulocyte function tests helped to focus attention on the monocyte, and Territo and Cline ${ }^{9}$ have described a group of monocyte function tests which are both simple and reproducible. One of the particular difficulties of testing monocyte function has been getting relatively pure suspensions of monocytes; Territo and Cline have separated monocytes from granulocytes with a Ficoll-Hypaque density gradient. Lymphocytes are then removed by adhesion to the surface of a plastic Petri dish and repeatedly washed. Using some of the principles established in tests of granulocyte function and some new techniques, they have devised methods $^{9}$ for measuring chemotaxis, adhesion, spreading, and phagocytosis. To evaluate fungal and bacterial killing they used Candida pseudotropicalis, which is killed by a myeloperoxidase-independent mechanism, and $C$ albicans, which requires myeloperoxidase for effective killing. Both systems need to be tested, since myeloperoxidase is lost as the human monocyte develops into the mature macrophage. Territo and Cline claim that their tests give basic information about the state of the host's defences and about the degree of monocyte activation and yet can be performed in any haematology laboratory. Other monocyte functions can be measured, including cell surface receptors, tumour killing and cytotoxicity, enzyme activity, and pinocytosis; but for the time 
being these will remain the province of the research worker.

Measurement of adhesion and spreading in macrophages appears to reflect phagocytic potential, and in animals these functions increase with macrophage activation. ${ }^{10}$ Tests of chemotaxis, phagocytosis, and micro-organism killing have been shown to be abnormal in a variety of conditions. For example, monocyte chemotaxis is defective in patients with mucocutaneous candidiasis ${ }^{11}$ and some carcinomas ${ }^{12}$ and in the Chediak-Higashi syndrome. ${ }^{13}$ There are reports of reduced monocyte microbial activity in chronic granulomatous disease, ${ }^{14}$ chronic neutropenia, ${ }^{15}$ and myeloperoxidase deficiency, ${ }^{16}$ and in patients receiving high-dose corticosteroid treatment. ${ }^{17}$ Monocyte phagocytosis is depressed in some patients with Hodgkin's disease and lymphosarcoma. ${ }^{18}$ Others have found that monocytes from most patients with myelomonocytic leukaemia and some patients with lymphoma phagocytose bacteria normally but do not kill intracellular bacteria. ${ }^{19}$

For the moment, monocyte function tests may not be of much direct benefit to patient care. Nevertheless, further defects associated with disease states will emerge only as these tests become more reliable and are more widely applied.

${ }^{1}$ Metchnikoff, E, Immunity in Infective Diseases. London, Cambridge University Press, 1905.

2 Aschoff, L, Lectures on Pathology. New York, Hoeber, 1924.

3 Lewis, M R, American fournal of Pathology, 1925, 1, 91.

4 Ebert, R H, and Florey, H W, British fournal of Experimental Pathology, $1939,20,342$.

5 Evans, R, and Alexander, P, Nature, 1972, 236, 168.

6 Hibbs, J B, Lambert, L H, and Remington, J S, Science, 1972, 177, 998.

${ }^{7}$ Chervenick, P A, and Lo Buglio, A F, Science, 1972, 178, 164.

${ }^{8}$ Golde, D W, and Cline, M J, fournal of Clinical Investigation, 1972, 51, 2981.

9 Territo, M C, and Cline, M J, fournal of Immunology, 1977, 118, 187

10 David, J R, Federation Proceedings, 1975, 34, 1730.

11 Snyderman, R L C, et al, Annals of Internal Medicine, 1973, 78, 509.

12 Snyderman, R, and Stahl, C, in The Phagocytic Cell in Host Resistance, eds J A Bellanti and D H Dayton. New York, Raven Press, 1975.

13 Gallin, J I, et al, Blood, 1975, 45, 863.

14 Davis, W C, et al, fournal of Immunology, 1968, 101, 1093.

15 Lehrer, R I, and Cline, M J, fournal of Clinical Investigation, 1969, 48, 1478.

${ }^{16}$ Baehner, R L, and Johnston, R B, Blood, 1972, 40, 31.

17 Rinehart, J J, et al, New England fournal of Medicine, 1975, 292, 236.

18 Ghosh, M L, Hudson, G, and Blackburn, E K, British fournal of Haematology, 1973, 25, 293.

${ }^{19}$ Cline, M J, fournal of Clinical Investigation, 1973, 52, 2185.

\section{Bigger outlet for the bile duct?}

Surgeons are all too familiar with the problem of residual stones in the common bile duct. Once the decision is made to explore the common duct there are two aims: to remove all of the stones and to prevent further stone formation.

Most often the bile duct is explored by an opening in the supraduodenal portion, but this practice is mechanically unsound. Clearly, the most effective way of removing particulate matter from a narrow tube is to open one end rather than making a hole half way along it. Exploration of the biliary ducts through the ampulla of Vater has its exponents, but they remain a minority, ${ }^{1}$ probably because the common duct is much easier to find and enter above the duodenum.

After they have removed stones from the common bile duct many surgeons rely on the passage of bougies and saline irrigation to ensure complete clearance. Unfortunately, using these methods alone, they will overlook stones in up to $10 \%$ of patients. ${ }^{2}$ Two ways are available for confirming that the duct is completely clear while the patient is still on the operating table. With careful technique ${ }^{3}$ useful postexploratory cholangiograms (which are worth while even though they are not as accurate as pre-exploratory films) can usually be obtained, though the contrast may fail to enter the duodenum simply as a result of the passage of bougies through the ampulla of Vater. More recently, direct examination of the duct with a choledochoscope has proved an effective way of finding missed stones. ${ }^{4}$

If despite repeated manipulation and irrigation calculi cannot be dislodged either from the intrahepatic ducts or from the ampulla of Vater, a duct drainage procedure may allow stones to pass later without causing symptoms. A duct drainage operation is also needed for patients with grossly dilated bile ducts, those whose ducts are full of mud or infected bile, and those (often found in the Far East) who have suppurative cholangiohepatitis-such patients may develop further stones and recurring symptoms even after having the duct opened and all stones removed. An operation that improves drainage of the bile from the common bile duct should prevent recurrent cholangitis.

Patients with grossly dilated bile ducts form the largest group needing a duct drainage procedure. They are often elderly; the duct frequently measures more than $20 \mathrm{~mm}$ in diameter, and yet often no mechanical obstruction can be found at the lower end. Sometimes the duct is so stretched that it becomes tortuous and the supraduodenal part lies horizontally. A side-to-side choledochoduodenostomy is the ideal operation for such patients. Provided the stoma is more than $25 \mathrm{~mm}$ in diameter further attacks of cholangitis are uncommon. ${ }^{5}$ In a collected series of 1255 operations recurrent cholangitis was recorded in only five cases. ${ }^{6} \mathrm{~A}$ possible objection to the operation is that the distal duct remains undrained, so that vegetable debris may accumulate and lead to infection, further stone formation, and even pancreatitis. In most of the documented series trouble with this "blind pouch" was not recorded unless the stoma was made too small. ${ }^{78}$

In those patients with only moderate bile duct dilatation (up to $15 \mathrm{~mm}$ in diameter) choledochoduodenostomy is less easy to perform satisfactorily because it is difficult to create an adequate stoma. In such patients a sphincteroplasty is a satisfactory alternative. ${ }^{910}$ The duodenum must be adequately mobilised so that it can be brought up into the wound. A fine bougie may be passed down from above to aid recognition of the ampulla and the duodenum can then be opened through a transverse incision that allows closure without any tension. The sphincter is incised superolaterally and the bile duct mucosa sutured to the duodenal mucosa to prevent later stenosis. The incision of the sphincter is continued upwards until the dilated lumen of the bile duct is reached. Complete clearance of stones from the distal duct is then easily confirmed.

The published results of sphincteroplasty are generally good. In a collected series of 1061 patients only seven had postoperative cholangitis. ${ }^{9}$ One study compared sphincteroplasty with choledochoduodenostomy and found the two operations equally effective, but the mortality rate of sphincteroplasty $(4.6 \%)$ was slightly higher than that of choledochoduodenostomy ( $3 \%$ ).

In summary, any surgeon doing a choledochotomy should consider whether an additional duct drainage procedure is indicated. If it is, then choledochoduodenostomy (for those with grossly dilated ducts) or sphincteroplasty will give very satisfactory results. 